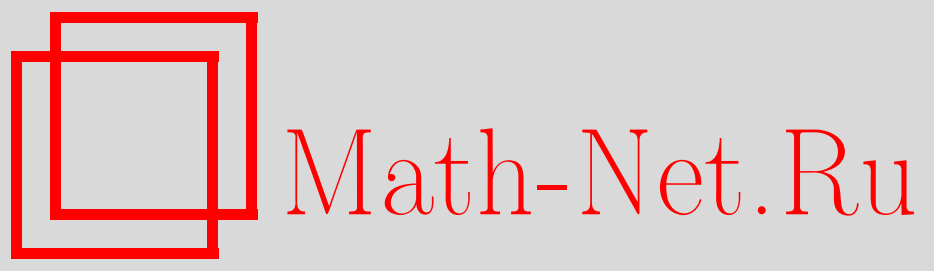

А. А. Серов, Образы конечного множества при итерациях двух случайных зависимых отображений, Дискрет. матем., 2015, том 27, выпуск 4, 133-140

DOI: https://doi.org/10.4213/dm1352

Использование Общероссийского математического портала Math-Net.Ru подразумевает, что вы прочитали и согласны с пользовательским соглашением http://www.mathnet.ru/rus/agreement

Параметры загрузки:

IP: 35.173 .219 .12

26 апреля 2023 г., 17:59:49

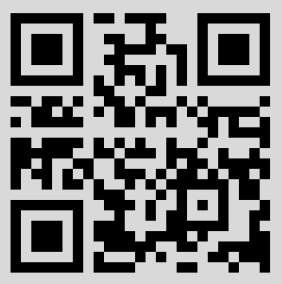




\title{
Образы конечного множества при итерациях двух случайных зависимых отображений
}

\author{
(c) 2015 г. A. A. CepoB*
}

Пусть $\mathcal{N}$ - множество из $N$ элементов и $\left(F_{1}, G_{1}\right),\left(F_{2}, G_{2}\right), \ldots-$ последовательность независимых пар таких случайных зависимых отображений $\mathcal{N} \rightarrow \mathcal{N}$, что $F_{k}$ и $G_{k}$ - случайные равновероятные отображения и $\mathbf{P}\left\{F_{k}(x)=G_{k}(x)\right\}=\alpha$ для любого $x \in \mathcal{N}$ и всех $k=1,2, \ldots$ Для подмножества $S_{0} \subset \mathcal{N},\left|S_{0}\right|=n$, рассматриваются последовательности его образов $S_{k}=F_{k}\left(\ldots F_{2}\left(F_{1}\left(S_{0}\right)\right) \ldots\right)$, $T_{k}=G_{k}\left(\ldots G_{2}\left(G_{1}\left(S_{0}\right)\right) \ldots\right), k=1,2 \ldots$, и последовательности их объединений $S_{k} \cup T_{k}$ и пересечений $S_{k} \cap T_{k}, k=1,2 \ldots$ Получены двусторонние неравенства для $\mathbf{M}\left|S_{k} \cup T_{k}\right|$ и $\mathbf{M}\left|S_{k} \cap T_{k}\right|$, в которых верхние оценки асимптотически эквивалентны нижним, если $N, n, k \rightarrow \infty, n k=o(N)$ и $\alpha=O\left(\frac{1}{N}\right)$.

Исследование выполнено за счет гранта Российского научного фонда (проект № 14-50-00005).

Ключевые слова: случайные отображения конечных множеств, совместные распределения, итерации случайных отображений, цепи Маркова.

\section{1. Введение}

Свойства случайных отображений конечных множеств изучаются уже несколько десятилетий (см., например, [4-9]). Как правило, рассматриваются свойства равновероятных случайных отображений и их итераций. В настоящей работе получены первые результаты о поведении образов конечного множества при параллельном применении к нему итераций двух зависимых случайных равновероятных отображений.

Пусть $\mathcal{N}=\{1, \ldots, N\}-$ конечное множество и $S_{0}=T_{0} \subseteq \mathcal{N},\left|S_{0}\right|=\left|T_{0}\right|=n-$ начальное подмножество. Пусть $\left(F_{1}, G_{1}\right),\left(F_{2}, G_{2}\right), \ldots$ - независимые пары случайных отображений множества $\mathcal{N}$ в себя, распределения которых для $x, y, z \in \mathcal{N}, y \neq z$, $k=1,2, \ldots$, определяются условиями

$$
\mathbf{P}\left\{F_{k}(x)=G_{k}(x)=y\right\}=\frac{\alpha}{N}, \quad \mathbf{P}\left\{F_{k}(x)=y, G_{k}(x)=z\right\}=\frac{1-\alpha}{N(N-1)},
$$

пары случайных величин $\left(F_{k}(x), G_{k}(x)\right), x \in \mathcal{N}$, предполагаются независимыми в совокупности. Таким образом, $F_{k}$ и $G_{k}$ - случайные равновероятные отображения

* Место работы: Математический институт им. В.А. Стеклова Российской академии наук, e-mail: serov@mi.ras.ru 
и

$$
\mathbf{P}\left\{F_{k}(x)=G_{k}(x)\right\}=\alpha, \quad \mathbf{P}\left\{F_{k}(x) \neq G_{k}(x)\right\}=1-\alpha, \quad x \in \mathcal{N}, k=1,2, \ldots
$$

Рассматриваются последовательности образов $S_{0}=T_{0}$ при последовательном применении к этому подмножеству отображений $F_{k}, G_{k}$ :

$$
\begin{gathered}
S_{1}=F_{1}\left(S_{0}\right), S_{2}=F_{2}\left(F_{1}\left(S_{0}\right)\right), \ldots, S_{t}=F_{t}\left(F_{t-1}\left(\ldots\left(F_{1}\left(S_{0}\right)\right) \ldots\right)\right) \\
T_{1}=G_{1}\left(T_{0}\right), T_{2}=G_{2}\left(G_{1}\left(T_{0}\right)\right), \ldots, T_{t}=G_{t}\left(G_{t-1}\left(\ldots\left(G_{1}\left(T_{0}\right)\right) \ldots\right)\right)
\end{gathered}
$$

Наша цель - получить оценки математических ожиданий чисел элементов в множествах $S_{k} \cup T_{k}, S_{k} \cap T_{k}, k=1,2, \ldots$ Оценки для $\left|S_{k}\right|$ были получены в [4] комбинаторно-вероятностными методами, здесь используется аппарат цепей Маркова.

\section{2. Основные результаты}

Теорема 1. Пусть $F_{1}, F_{2}, \ldots u G_{1}, G_{2}, \ldots$ - независимые равновероятные отображения множества $\mathcal{N}=\{1, \ldots, N\}$ в себя, удовлетворяющие условиям (1) $\frac{1}{N} \leqslant \alpha<1$, пусть $S_{k} u T_{k}, k=1,2, \ldots,-$ последовательности образов множества $S_{0} \subseteq \mathcal{N},\left|S_{0}\right|=n$, определенные формулами (2), u $\lambda_{1}=1-\frac{1}{N}, \lambda_{2}=\alpha-\frac{1}{N}$, $\lambda_{3}=\left(1-\frac{2}{N}\right)\left(\alpha-\frac{1}{N}\right)$. Для любого элемента $x \in \mathcal{N}$, не зависящего от отображений $F_{1}, F_{2}, \ldots$ и $G_{1}, G_{2}, \ldots$, при любъх $1 \leqslant k, n \leqslant N$ справедливы двусторонние оченки

$$
L(k, N) \leqslant \mathbf{P}\left\{x \in S_{k} \cup T_{k}|| S_{0} \mid=n\right\}<L(k, N)+U(k, N),
$$

где

$$
\begin{gathered}
L(k, N)=\frac{n}{N} \cdot \frac{1+N\left(\lambda_{1}-\lambda_{2}\right)\left(2-\lambda_{2}^{k}\right)}{N\left(1-\lambda_{2}\right)}-\frac{n(n-1)}{N}\left(1-\lambda_{1}^{k}+\frac{1}{N} \frac{1-\lambda_{2}^{k}}{1-\lambda_{2}}\right), \\
U(k, N)=\frac{n(n-1)}{N^{2}}\left(\frac{(n-2) k^{2}}{2 N}+\frac{n\left(1-\lambda_{1}^{k}\right)}{1-\lambda_{2}}+\frac{\lambda_{2}\left(\lambda_{1}^{k} n-\lambda_{3}^{k}(n-2)\right)}{\left(N\left(\lambda_{1}-\lambda_{2}\right)+2 \lambda_{2}\right)\left(1-\lambda_{2}\right)}+\frac{\left(\lambda_{2}^{k}-\lambda_{3}^{k}\right) N\left(\lambda_{1}-\lambda_{2}\right)}{1-\lambda_{2}}\right) .
\end{gathered}
$$

Утверждение 1. При $\alpha=1$ последовательности $F_{1}, F_{2}, \ldots u G_{1}, G_{2}, \ldots$ совпадаюо $u$

$$
\frac{n}{N}-C_{n}^{2} \frac{1-\lambda_{1}^{k}}{N} \leqslant \mathbf{P}\left\{x \in S_{k}|| S_{0} \mid=n\right\}<\frac{n}{N}-C_{n}^{2} \frac{1-\lambda_{1}^{k}}{N}+C_{n}^{3} \frac{3 k^{2}}{2 N^{3}} .
$$

Следствие 1. При $\frac{1}{N} \leqslant \alpha<1$ справедливы также следующие ощенки:

$$
\begin{gathered}
L(k, N) N \leqslant \mathbf{M}\left\{\left|S_{k} \cup T_{k}\right||| S_{0} \mid=n\right\}<(L(k, N)+U(k, N)) N, \\
2 n-2 C_{n}^{2}\left(1-\lambda_{1}^{k}\right)-(L(k, N)+U(k, N)) N<\mathbf{M}\left\{\left|S_{k} \cap T_{k}\right||| S_{0} \mid=n\right\}< \\
<2 n-2 C_{n}^{2}\left(1-\lambda_{1}^{k}\right)+3 C_{n}^{3} \frac{k^{2}}{N^{2}}-L(k, N) N .
\end{gathered}
$$

Доказательство теоремы 1. Будем использовать обозначения $F_{k \ldots 1}(x), G_{k \ldots 1}(x)$ для $F_{k}\left(\ldots\left(F_{1}(x)\right) \ldots\right), G_{k}\left(\ldots\left(F_{1}(x)\right) \ldots\right)$ соответственно. Из равновероятности отображений $F_{j}, G_{j}, j \geqslant 1$, следует, что величина $\mathbf{P}\left\{x \in\left\{S_{k} \cup T_{k}\right\}|| S_{0} \mid=n\right\}$ не зависит от $x \in \mathcal{N}$ как при фиксированном $x$, так и при случайном $x$, принимающем значения 
в $\mathcal{N}$ и не зависящем от $F_{1}, F_{2}, \ldots$ и $G_{1}, G_{2}, \ldots$ Поэтому неравенства $(3)$ достаточно доказать для $x=1$. Так как

$$
\left\{1 \in S_{k} \cup T_{k}\right\}=\bigcup_{x \in S_{0}}\left(\left\{F_{k \ldots 1}(x)=1\right\} \cup\left\{G_{k \ldots 1}(x)=1\right\}\right)
$$

и распределения пар $\left(F_{k}, G_{k}\right)$ симметричны, то вероятности событий, различающихся только перестановками символов $F$ и $G$, одинаковы; с учетом этого неравенства Бонферрони для вероятности объединения событий в нашем случае принимают вид

$$
\begin{aligned}
& L(k, N) \stackrel{\text { def }}{=} 2 \sum_{x \in S_{0}} \mathbf{P}\left\{F_{k \ldots 1}(x)=1\right\}-2 \sum_{\substack{x, y \in S_{0} \\
x<y}} \mathbf{P}\left\{F_{k \ldots 1}(x)=F_{k \ldots 1}(y)=1\right\}- \\
& -\sum_{x, y \in S_{0}} \mathbf{P}\left\{F_{k \ldots 1}(x)=G_{k \ldots 1}(y)=1\right\} \leqslant \mathbf{P}\left\{1 \in S_{k} \cup T_{k}|| S_{0} \mid=n\right\} \leqslant \\
& \leqslant L(k, N)+2 \sum_{\substack{x, y, z \in S_{0} \\
x<y<z}} \mathbf{P}\left\{F_{k \ldots 1}(x)=F_{k \ldots 1}(y)=F_{k \ldots 1}(z)=1\right\}+ \\
& +2 \sum_{\substack{x, y, z \in S_{0} \\
x<y}} \mathbf{P}\left\{F_{k \ldots 1}(x)=F_{k \ldots 1}(y)=G_{k \ldots 1}(z)=1\right\} \stackrel{\text { def }}{=} L(k, N)+U(k, N) .
\end{aligned}
$$

Так как отображения $F_{1}, F_{2}, \ldots$ и $G_{1}, G_{2}, \ldots$ равновероятны, то $\mathbf{P}\left\{F_{k \ldots 1}(x)=1\right\}=$ $\mathbf{P}\left\{G_{k \ldots 1}(x)=1\right\}=\frac{1}{N}$ для любого $x \in \mathcal{N}$ и поэтому

$$
\sum_{x \in S_{0}} \mathbf{P}\left\{F_{k \ldots 1}(x)=1\right\}=\frac{n}{N}
$$

Далее, при любых $x, y \in \mathcal{N}, x \neq y$, см. [4, формула (10), с. 47],

$$
\mathbf{P}\left\{F_{k \ldots 1}(x)=F_{k \ldots 1}(y)=1\right\}=\frac{1}{N}\left(1-\left(1-\frac{1}{N}\right)^{k}\right) \in\left[\frac{k}{N^{2}}-C_{k}^{2} \frac{1}{N^{2}}, \frac{k}{N^{2}}\right],
$$

поэтому

$$
\sum_{\substack{x, y \in S_{0} \\ x<y}} \mathbf{P}\left\{F_{k \ldots 1}(x)=F_{k \ldots 1}(y)=1\right\}=\frac{C_{n}^{2}}{N}\left(1-\left(1-\frac{1}{N}\right)^{k}\right) .
$$

Лемма 1. При $x, y \in \mathcal{N} u k=1,2, \ldots$ справедливы равенства

$$
\mathbf{P}\left\{F_{k \ldots 1}(x)=G_{k \ldots 1}(y)=1\right\}= \begin{cases}\frac{1}{N^{2}} \cdot \frac{1+N(1-\alpha)\left(\alpha-\frac{1}{N}\right)^{k}}{1-\left(\alpha-\frac{1}{N}\right)}, & \text { если } x=y, \\ \frac{1}{N^{2}} \cdot \frac{1-\left(\alpha-\frac{1}{N}\right)^{k}}{1-\left(\alpha-\frac{1}{N}\right)}, & \text { если } x \neq y .\end{cases}
$$

Доказательство. Из независимости пар $\left(F_{k}, G_{k}\right), k=1,2, \ldots$, и предположений $(2)$ следует, что при любых $x, y \in \mathcal{N}$ событие $\left\{F_{k+1 \ldots 1}(x)=G_{k+1 \ldots 1}(y)\right\}=$ $\left\{F_{k+1}\left(F_{k \ldots 1}(x)\right)=G_{k+1}\left(G_{k \ldots 1}(x)\right)\right\}$ не зависит от значений $F_{j \ldots 1}(x), G_{j \ldots 1}(y)(1 \leqslant j<$ $k)$ и зависит лишь от того, выполняется или нет равенство $F_{k \ldots 1}(x)=G_{k \ldots 1}(x)$ :

$$
\begin{gathered}
\mathbf{P}\left\{F_{k+1 \ldots 1}(x)=G_{k+1 \ldots 1}(y) \mid F_{k \ldots 1}(x) \neq G_{k \ldots 1}(y)\right\}=\frac{1}{N} \\
\mathbf{P}\left\{F_{k+1 \ldots 1}(x)=G_{k+1 \ldots 1}(y) \mid F_{k \ldots 1}(x)=G_{k \ldots 1}(y)\right\}=\alpha .
\end{gathered}
$$


Поэтому при любых $x, y \in \mathcal{N}$ последовательность $\left\{\delta_{k}=\mathbb{I}\left\{F_{k \ldots 1}(x)=G_{k \ldots 1}(y)\right\}\right\}_{k=1}^{\infty}$ является цепью Маркова с множеством состояний $\{0,1\}$ и матрицей вероятностей переходов

$$
P_{2}=\left(\begin{array}{cc}
1-\frac{1}{N} & \frac{1}{N} \\
1-\alpha & \alpha
\end{array}\right)=\left(\begin{array}{cc}
\frac{1}{2}+\left(\frac{1}{2}-\frac{1}{N}\right) & \frac{1}{2}-\left(\frac{1}{2}-\frac{1}{N}\right) \\
\frac{1}{2}-\left(\alpha-\frac{1}{2}\right) & \frac{1}{2}+\left(\alpha-\frac{1}{2}\right)
\end{array}\right)
$$

так как $\mathbf{P}\left\{F_{k \ldots 1}(x)=G_{k \ldots 1}(y)=1 \mid F_{k \ldots 1}(x)=G_{k \ldots 1}(y)\right\}=\frac{1}{N}$ в силу равновероятности отображений $F_{k}$, то

$$
\begin{gathered}
\mathbf{P}\left\{F_{k \ldots 1}(x)=G_{k \ldots 1}(x)=1\right\}=\frac{1}{N} \mathbf{P}\left\{\delta_{k}=1 \mid \delta_{0}=1\right\}, \\
\mathbf{P}\left\{F_{k \ldots 1}(x)=G_{k \ldots 1}(y)=1\right\}=\frac{1}{N} \mathbf{P}\left\{\delta_{k}=1 \mid \delta_{0}=0\right\}, x \neq y .
\end{gathered}
$$

Утверждение 2. Если $u+v \neq 1 u Q=\left(\begin{array}{cc}\frac{1}{2}+u & \frac{1}{2}-u \\ \frac{1}{2}-v & \frac{1}{2}+v\end{array}\right)$, то при любом иелом $k=1,2, \ldots$

$$
Q^{k}=\left(\begin{array}{cc}
\frac{1-2 v+(1-2 u)(u+v)^{k}}{2(1-u-v)} & \frac{(1-2 u)\left(1-(u+v)^{k}\right)}{2(1-u-v)} \\
\frac{(1-2 v)\left(1-(u+v)^{k}\right)}{2(1-u-v)} & \frac{1-2 u+(1-2 v)(u+v)^{k}}{2(1-u-v)}
\end{array}\right) .
$$

Доказательство утверждения 2. Утверждение 2 можно доказать индукцией по $k$. При $k=0$ утверждение верно. Так как $Q-$ стохастическая матрица, то $Q^{k}-$ стохастическая при любом целом $k \geqslant 1$, т. е. сумма двух элементов каждой строки равна 1; поэтому достаточно доказать справедливость формулы для диагональных элементов. Ввиду симметричности записи элементов матрицы $Q$ шаг индукции достаточно провести для элемента в левом верхнем углу:

$$
\begin{aligned}
& \left(\frac{1}{2}+u\right)\left(1-2 v+(1-2 u)(u+v)^{k}\right)+\left(\frac{1}{2}-u\right)\left(1-2 v-(1-2 v)(u+v)^{k}\right)= \\
& =1-2 v+\frac{1-2 u}{2}(1+2 u-(1-2 v))(u+v)^{k}=1-2 v+(1-2 u)(u+v)^{k+1} .
\end{aligned}
$$

Утверждение Леммы 1 непосредственно следует из формул (11), (13) и (12).

Из Леммы 1 следует, что

$$
\begin{gathered}
\sum_{x, y \in S_{0}} \mathbf{P}\left\{F_{k \ldots 1}(x)=G_{k \ldots 1}(y)=1\right\}= \\
=\sum_{x \in S_{0}} \mathbf{P}\left\{F_{k \ldots 1}(x)=G_{k \ldots 1}(x)=1\right\}+\sum_{\substack{x, y \in S_{0} \\
x \neq y}} \mathbf{P}\left\{F_{k \ldots 1}(x)=G_{k \ldots 1}(y)=1\right\}= \\
=\frac{n}{N} \cdot \frac{1+N(1-\alpha)\left(\alpha-\frac{1}{N}\right)^{k}}{1+N(1-\alpha)}+\frac{n(n-1)}{N^{2}} \cdot \frac{1-\left(\alpha-\frac{1}{N}\right)^{k}}{1-\left(\alpha-\frac{1}{N}\right)} .
\end{gathered}
$$

Отсюда и из равенств $(7),(8),(9)$ следует, что

$$
\begin{aligned}
\mathbf{P}\left\{1 \in S_{k} \cup T_{k}|| S_{0} \mid=n\right\} \geqslant L(k, N)= \\
\geqslant \frac{2 n}{N}-\frac{n(n-1)}{N}\left(1-\left(1-\frac{1}{N}\right)^{k}+\frac{1-\left(\alpha-\frac{1}{N}\right)^{k}}{1+N(1-\alpha)}\right)-\frac{n}{N} \cdot \frac{1+N(1-\alpha)\left(\alpha-\frac{1}{N}\right)^{k}}{1+N(1-\alpha)}= \\
\quad=\frac{n}{N} \cdot \frac{1+N(1-\alpha)\left(2-\left(\alpha-\frac{1}{N}\right)^{k}\right)}{1+N(1-\alpha)}-\frac{n(n-1)}{N}\left(1-\left(1-\frac{1}{N}\right)^{k}+\frac{1-\left(\alpha-\frac{1}{N}\right)^{k}}{1+N(1-\alpha)}\right) .
\end{aligned}
$$


Заменяя здесь $1-\frac{1}{N}$ на $\lambda_{1}$ и $1+N(1-\alpha)$ на $N\left(1-\lambda_{2}\right)$, получаем левое неравенство в (3).

Точные значения слагаемых в суммах в правой части неравенства Бонферрони (7) содержатся в леммах 2 и 3.

Лемма 2. Если $x, y, z \in \mathcal{N}$ попарно различнъ, то

$$
\mathbf{P}\left\{F_{k \ldots 1}(x)=F_{k \ldots 1}(y)=F_{k \ldots 1}(z)=1\right\}=\frac{1}{N}\left(1-\frac{1}{2}\left(1-\frac{1}{N}\right)^{k}\left(3-\left(1-\frac{2}{N}\right)^{k}\right)\right) .
$$

Доказательство. Последовательность $\left\{\left(F_{k \ldots 1}(x), F_{k \ldots 1}(y), F_{k \ldots 1}(z)\right)\right\}$, очевидно, образует цепь Маркова, так как отображения $F_{1}, F_{2}, \ldots$ независимы. Из равновероятности отображений $F_{1}, F_{2}, \ldots$ следует, что множество состояний этой цепи можно укрупнить, сохраняя марковость: последовательность $\zeta_{k}=\mid\left\{F_{k \ldots 1}(x)\right\} \cup\left\{F_{k \ldots 1}(y)\right\} \cup$ $\left\{F_{k \ldots 1}(z)\right\} \mid$ образует цепь Маркова с состояниями $1,2,3$, и

$$
\mathbf{P}\left\{F_{k \ldots 1}(x)=F_{k \ldots 1}(y)=F_{k \ldots 1}(z)\right\}=\mathbf{P}\left\{\zeta_{k}=1 \mid \zeta_{0}=3\right\}
$$

так что

$$
\mathbf{P}\left\{F_{k \ldots 1}(x)=F_{k \ldots 1}(y)=F_{k \ldots 1}(z)=1\right\}=\frac{1}{N} \mathbf{P}\left\{\zeta_{k}=1 \mid \zeta_{0}=3\right\} .
$$

Легко проверить, что матрица переходных вероятностей цепи $\left\{\zeta_{k}\right\}$ имеет вид

$$
P_{3}=\left(\begin{array}{ccc}
1 & 0 & 0 \\
\frac{1}{N} & 1-\frac{1}{N} & 0 \\
\frac{1}{N^{2}} & \left(1-\frac{1}{N}\right) \frac{3}{N} & \left(1-\frac{1}{N}\right)\left(1-\frac{2}{N}\right)
\end{array}\right) \text {. }
$$

Индукцией по $k$ доказывается следующая формула (для компактности матрица $P_{3}^{k}$ записана в транспонированном виде):

$$
\left(P_{3}^{k}\right)^{T}=\left(\begin{array}{ccc}
1 & 1-\left(1-\frac{1}{N}\right)^{k} & 1-\frac{1}{2}\left(1-\frac{1}{N}\right)^{k}\left(3-\left(1-\frac{2}{N}\right)^{k}\right) \\
0 & \left(1-\frac{1}{N}\right)^{k} & \frac{3}{2}\left(1-\frac{1}{N}\right)^{k}\left(1-\left(1-\frac{2}{N}\right)^{k}\right) \\
0 & 0 & \left(\left(1-\frac{1}{N}\right)\left(1-\frac{2}{N}\right)\right)^{k}
\end{array}\right)^{T}
$$

Лемма 2 доказана.

Отметим, что в $[4$, формула (11), с. 48]) для попарно различных $x, y, z \in \mathcal{N}$ другим методом было доказано неравенство

$$
\mathbf{P}\left\{F_{k \ldots 1}(x)=F_{k \ldots 1}(y)=F_{k \ldots 1}(z)=1\right\} \leqslant \frac{3 k^{2}}{2 N^{3}} .
$$


Лемма 3. При $\lambda_{1}=1-\frac{1}{N}, \lambda_{2}=\alpha-\frac{1}{N}, \lambda_{3}=\left(1-\frac{2}{N}\right)\left(\alpha-\frac{1}{N}\right)$ и попарно различных $x, y, z \in \mathcal{N}$ справедливы равенства

$$
\begin{gathered}
\mathbf{P}\left\{F_{k \ldots 1}(x)=F_{k \ldots 1}(y)=G_{k \ldots 1}(z)=1\right\}= \\
=\frac{1}{N^{2}\left(1-\lambda_{2}\right)}\left(1-\lambda_{1}^{k}-\lambda_{2}^{k}+\lambda_{3}^{k}+\frac{\left(\lambda_{1}^{k}-\lambda_{3}^{k}\right) \lambda_{2}}{N\left(\lambda_{1}-\lambda_{2}\right)+2 \lambda_{2}}\right), \\
\mathbf{P}\left\{F_{k \ldots 1}(x)=F_{k \ldots 1}(y)=G_{k \ldots 1}(x)=1\right\}= \\
=\mathbf{P}\left\{F_{k \ldots 1}(x)=F_{k \ldots 1}(y)=G_{k \ldots 1}(y)=1\right\}= \\
=\frac{1}{N^{2}\left(1-\lambda_{2}\right)}\left(1-\lambda_{1}^{k}-\frac{1}{2} \lambda_{2}^{k}+\frac{N\left(\lambda_{2}-\lambda_{1}\right)\left(\lambda_{3}^{k}-\lambda_{2}^{k}\right)}{2}+\frac{\frac{1}{2} N\left(\lambda_{1}-\lambda_{2}\right) \lambda_{3}^{k}+\lambda_{1}^{k} \lambda_{2}}{N\left(1-\lambda_{2}\right)+2 \lambda_{2}-1}\right) .
\end{gathered}
$$

Доказательство. Лемма 3 , как и лемма 2, доказывается с помощью аппарата цепей Маркова. Последовательность $\left\{\left(F_{k \ldots 1}(x), F_{k \ldots 1}(y), G_{k \ldots 1}(z)\right)\right\}$ образует цепь Маркова, так как пары отображений $\left(F_{1}, G_{1}\right),\left(F_{2}, G_{2}\right), \ldots$ независимы.

Из равновероятности отображений следует, что множество состояний этой цепи можно укрупнить, сохраняя марковость. Занумеруем состояния укрупненной цепи $\left\{\gamma_{t}\right\}$ следующим образом:

$$
\begin{array}{ccc}
\ll 1 »: & \left|\left\{F_{k \ldots 1}(x)\right\} \cup\left\{F_{k \ldots 1}(y)\right\} \cup\left\{G_{k \ldots 1}(z)\right\}\right|=3, \\
\ll 2 »: & \left.\left|\left\{F_{k \ldots 1}(x)\right\} \cup\left\{F_{k \ldots 1}(y)\right\} \cup\left\{G_{k \ldots 1}(z)\right\}\right|=\mid\left\{F_{k \ldots 1}(x)\right\} \cup\left\{F_{k \ldots 1}(y)\right\}\right\} \mid=2, \\
\ll 3 »: & \left.\left|\left\{F_{k \ldots 1}(x)\right\} \cup\left\{F_{k \ldots 1}(y)\right\} \cup\left\{G_{k \ldots 1}(z)\right\}\right|=2, \mid\left\{F_{k \ldots 1}(x)\right\} \cup\left\{F_{k \ldots 1}(y)\right\}\right\} \mid=1, \\
\ll 4 »: & \left|\left\{F_{k \ldots 1}(x)\right\} \cup\left\{F_{k \ldots 1}(y)\right\} \cup\left\{G_{k \ldots 1}(z)\right\}\right|=1
\end{array}
$$

Матрица переходных вероятностей цепи $\left\{\gamma_{t}\right\}$ имеет вид

$$
P_{4}=\left(\begin{array}{cccc}
\left(1-\frac{1}{N}\right)\left(1-\frac{2}{N}\right) & \left(1-\frac{1}{N}\right) \frac{2}{N} & \frac{1}{N}\left(1-\frac{1}{N}\right) & \frac{1}{N^{2}} \\
\left(1-\frac{1}{N}\right)(1-\alpha)\left(1-\frac{1}{N-1}\right) & \left(1-\frac{1}{N}\right)\left(\alpha+\frac{1-\alpha}{N-1}\right) & \frac{1-\alpha}{N} & \frac{\alpha}{N} \\
0 & 0 & \left(1-\frac{1}{N}\right) & \frac{1}{N} \\
0 & 0 & (1-\alpha) & \alpha
\end{array}\right) .
$$

Приведенные в лемме 3 формулы совпадают с вероятностями переходов цепи $\left\{\gamma_{t}\right\}$ из состояний 1 и 2 в состояние 4 .

Указанные в формулировке леммы числа $\lambda_{1}, \lambda_{2}, \lambda_{3}$ являются корнями характеристического многочлена матрицы $P_{4}$; правильность выражений элементов матриц $P_{4}^{k}$, полученных методами линейной алгебры ( $[3$, Гл. IV, §5]), проверялась с помощью систем символьных вычислений. Подробный вывод этих формул здесь не приводится ввиду большой громоздкости.

Утверждение 3. При $\lambda_{1}=1-\frac{1}{N}, \lambda_{2}=\alpha-\frac{1}{N} \geqslant 0, \lambda_{3}=\left(1-\frac{2}{N}\right)\left(\alpha-\frac{1}{N}\right)$ u попарно различных $x, y, z \in \mathcal{N}$ справедливы оченки

$$
\begin{aligned}
& \mathbf{P}\left\{F_{k \ldots 1}(x)=F_{k \ldots 1}(y)=G_{k \ldots 1}(z)=1\right\} \leqslant \\
& \leqslant \frac{1}{N^{2}\left(1-\lambda_{2}\right)}\left(1-\lambda_{1}^{k}+\frac{\lambda_{2}\left(\lambda_{1}^{k}-\lambda_{3}^{k}\right)}{N\left(\lambda_{1}-\lambda_{2}\right)+2 \lambda_{2}}\right), \\
& \mathbf{P}\left\{F_{k \ldots 1}(x)=F_{k \ldots 1}(y)=G_{k \ldots 1}(x)=1\right\} \leqslant \\
& \leqslant \frac{1}{N^{2}\left(1-\lambda_{2}\right)}\left(1-\lambda_{1}^{k}\left(1-\frac{\lambda_{2}}{N\left(\lambda_{1}-\lambda_{2}\right)+2 \lambda_{2}}\right)+\frac{1}{2}\left(\lambda_{2}^{k}-\lambda_{3}^{k}\right) N\left(\lambda_{1}-\lambda_{2}\right)\right) .
\end{aligned}
$$


Доказательство. Из (17) с учетом того, что $\lambda_{3}=\frac{N-2}{N} \lambda_{2}$, получаем оценку

$$
\mathbf{P}\left\{F_{k \ldots 1}(x)=F_{k \ldots 1}(y)=G_{k \ldots 1}(z)=1\right\} \leqslant \frac{1}{N^{2}\left(1-\lambda_{2}\right)}\left(1-\lambda_{1}^{k}+\frac{\lambda_{2}\left(\lambda_{1}^{k}-\lambda_{3}^{k}\right)}{N\left(\lambda_{1}-\lambda_{2}\right)+2 \lambda_{2}}\right) .
$$

Из (18) и $\lambda_{2} \geqslant 0$ следует, что при $x \neq y$

$$
\begin{gathered}
\mathbf{P}\left\{F_{k \ldots 1}(x)=F_{k \ldots 1}(y)=G_{k \ldots 1}(x)=1\right\}= \\
=\frac{1}{N^{2}\left(1-\lambda_{2}\right)}\left(1-\lambda_{1}^{k}-\frac{1}{2} \lambda_{2}^{k}+\frac{1}{2} N\left(\lambda_{2}-\lambda_{1}\right)\left(\lambda_{3}^{k}-\lambda_{2}^{k}\right)+\frac{\frac{1}{2} N\left(\lambda_{1}-\lambda_{2}\right) \lambda_{3}^{k}+\lambda_{1}^{k} \lambda_{2}}{N\left(\lambda_{1}-\lambda_{2}\right)+2 \lambda_{2}}\right) \leqslant \\
\leqslant \frac{1}{N^{2}\left(1-\lambda_{2}\right)}\left(1-\lambda_{1}^{k}\left(1-\frac{\lambda_{2}}{N\left(\lambda_{1}-\lambda_{2}\right)+2 \lambda_{2}}\right)+\frac{1}{2}\left(\lambda_{2}^{k}-\lambda_{3}^{k}\right)\left(N\left(\lambda_{1}-\lambda_{2}\right)-1\right)\right) .
\end{gathered}
$$

Учитывая, что $\lambda_{3}=\frac{N-2}{N} \lambda_{2}$, получим вторую оценку в (19).

Так как вероятности (17) и (18) входят в последнюю сумму верхней оценки (7) в точности $n(n-1)(n-2) / 2$ и $n(n-1)$ раз соответственно, то из $(7),(14),(16),(19)$ следует, что

$$
\begin{aligned}
& \mathbf{P}\left\{1 \in S_{k} \cup T_{k}|| S_{0} \mid=n\right\} \leqslant \\
& \leqslant L(k, N)+\frac{n(n-1)(n-2) k^{2}}{2 N^{3}}+\frac{n(n-1)(n-2)}{N^{2}\left(1-\lambda_{2}\right)}\left(1-\lambda_{1}^{k}+\frac{\lambda_{2}\left(\lambda_{1}^{k}-\lambda_{3}^{k}\right)}{N\left(\lambda_{1}-\lambda_{2}\right)+2 \lambda_{2}}\right)+ \\
& \quad+\frac{2 n(n-1)}{N^{2}\left(1-\lambda_{2}\right)}\left(1-\lambda_{1}^{k}\left(1-\frac{\lambda_{2}}{N\left(\lambda_{1}-\lambda_{2}\right)+2 \lambda_{2}}\right)+\frac{1}{2}\left(\lambda_{2}^{k}-\lambda_{3}^{k}\right) N\left(\lambda_{1}-\lambda_{2}\right)\right) .
\end{aligned}
$$

Тем самым второе неравенство в (3) и теорема доказаны.

Доказательство утверждения 1. Так как при $\alpha=1$ последовательности $F_{1}, F_{2}, \ldots$ и $G_{1}, G_{2}, \ldots$, очевидно, совпадают, то $S_{k}=T_{k}, k=1,2, \ldots$, и

$$
\left\{1 \in S_{k} \cup T_{k}\right\}=\left\{1 \in S_{k}\right\}=\bigcup_{x \in S_{0}}\left\{F_{k \ldots 1}(x)=1\right\} .
$$

Неравенства Бонферрони для вероятности такого события принимают вид

$$
\begin{gathered}
L^{*}(k, N) \stackrel{\text { def }}{=} \sum_{x \in S_{0}} \mathbf{P}\left\{F_{k \ldots 1}(x)=1\right\}-\sum_{\substack{x, y \in S_{0} \\
x<y}} \mathbf{P}\left\{F_{k \ldots 1}(x)=F_{k \ldots 1}(y)=1\right\} \leqslant \\
\leqslant \mathbf{P}\left\{1 \in S_{k}|| S_{0} \mid=n\right\} \leqslant \\
\leqslant L^{*}(k, N)+\sum_{\substack{x, y, z \in S_{0} \\
x<y<z}} \mathbf{P}\left\{F_{k \ldots 1}(x)=F_{k \ldots 1}(y)=F_{k \ldots 1}(z)=1\right\} .
\end{gathered}
$$

Из (8), (9), (16) и (21) следует (4).

Утверждение 1 доказано. 
Доказательство следствия 1. Так как

$$
\left|S_{k} \cup T_{k}\right|=\sum_{x \in \mathcal{N}} \mathbb{I}\left\{x \in S_{k} \cup T_{k}\right\}, \quad\left|S_{k} \cap T_{k}\right|=\sum_{x \in \mathcal{N}} \mathbb{I}\left\{x \in S_{k} \cap T_{k}\right\},
$$

то первое неравенство для математического ожидания (5) непосредственно следует из (3).

Далее, согласно утверждению 1 и равенству $\left|S_{k}\right|=\sum_{x \in \mathcal{N}} \mathbb{I}\left\{x \in S_{k}\right\}$, для математических ожиданий мощностей образов $S_{k}$ и $T_{k}$ справедлива двусторонняя оценка

$$
L^{*}(k, N) N \leqslant \mathbf{M}\left\{\left|S_{k}\right||| S_{0} \mid=n\right\}=\mathbf{M}\left\{\left|T_{k}\right||| S_{0} \mid=n\right\}<\left(L^{*}(k, N)+U^{*}(k, N)\right) N,
$$

где $L^{*}(k, N)=\frac{n}{N}-C_{n}^{2} \frac{1-\lambda_{1}^{k}}{N}, U^{*}(k, N)=C_{n}^{3} \frac{3 k^{2}}{2 N^{3}}$. Отсюда, учитывая тождества $\left|S_{k} \cap T_{k}\right|=\left|S_{k}\right|+\left|T_{k}\right|-\left|S_{k} \cup T_{k}\right|$, получаем оценки

$$
\begin{gathered}
2 L^{*}(k, N) N-(L(k, N)+U(k, N)) N \leqslant \mathbf{M}\left|S_{k} \cap T_{k}\right| \leqslant \\
2\left(L^{*}(k, N)+U^{*}(k, N)\right) N-L(k, N) N
\end{gathered}
$$

из которых следует (6).

Следствие 1 доказано.

Автор благодарит А.М. Зубкова за постановку задачи и постоянное внимание к работе.

\section{Список литературы}

1. Колчин В. Ф., Случайные отображения, М.: Наука, 1984, 208 с.

2. Колчин В. Ф., Севастьянов Б. А., Чистяков В. П., Случайные размещения, М. : Наука, $1976,224 \mathrm{c}$.

3. Гантмахер Ф. Р., Теория матрии, М.: «Наука», 1966, 576 с.

4. Зубков А.М., Серов А.А., "Совокупность образов подмножества конечного множества при итерациях случайных отображений”, Дискретная математика, 26:4 (2014), 43-50.

5. Степанов В. Е., “О распределении числа вершин в слоях случайного дерева", Теория вероятн. и примен., 14:1 (1969), 64-77.

6. Dalal A., Schmutz E. "Compositions of random functions on a finite set", Electr. J. Comb., 9:R26 (2002).

7. Flajolet P., Odlyzko A. M., "Random mapping statistics", Lect. Notes Comput. Sci., 434, 1990, 329-354.

8. Goh W. M. Y., Hitczenko P., Schmutz E., "Iterating random functions on a finite set", 2014, 7 pp., arXiv:math/0207276v2.

9. Kingman J. F. C., "The coalescent", Stoch.Proc. Appl., 13 (1982), 235-248.

Статья поступила 30.10.2015. 